\title{
MODELLING AND CONTROL OF CO-GENERATION POWER PLANTS UNDER CONSIDERATION OF LIFETIME CONSUMPTION: A HYBRID SYSTEM APPROACH
}

\author{
Giancarlo Ferrari-Trecate ${ }^{*, 1}$, Eduardo Gallestey ${ }^{* *}$, \\ Alec Stothert**, Geir Hovland ${ }^{* *}$, Paolo Letizia*, \\ Matteo Spedicato*, Manfred Morari*, Marc Antoine*** \\ * Institut für Automatik \\ ETH - Eidgenössische Technische Hochschule Zürich \\ CH 8092 Zürich \\ Tel. +411632 7626 Fax +411632 1211 \\ $\{$ ferrari, letizia, spedicat, morari\}@aut.ee.ethz.ch \\ ** ABB Corporate Research Ltd. \\ eduardo.gallestey, alec.stothert@ch.abb.com \\ *** ABB Power Automation Ltd. \\ marc.antoine@ch.abb.com
}

\begin{abstract}
In this paper the load optimization of a combined cycle power plant under consideration of the real cost of lifetime usage is accomplished by exploiting hybrid systems, i.e., systems evolving according to continuous dynamics, discrete dynamics, and logic rules. The possibility of turning on/off the gas and steam turbines, the operating constraints (minimum up and down times) and the different types of start up of the turbines characterize the hybrid behavior of a combined cycle power plant. In order to model both the continuous/discrete dynamics and the switching between different operating conditions we use the framework of Mixed Logic Dynamical systems. Next, we recast the economic optimization problem as a Model Predictive Control (MPC) problem, that allows us to optimize the plant operations by taking into account the time variability of both prices and electricity/steam demands. Because of the presence of integer variables, the MPC scheme is formulated as a mixed integer linear program that can be solved in an efficient way by using commercial solvers.
\end{abstract}

Keywords: Hybrid systems; Model predictive control; Combined cycle power plant; Mixed integer linear programming

\section{INTRODUCTION}

During the last two decades, the electric power industry has been subject to deep changes in structure and organization. From the technological side, the use of combined cycle power plants (CCPP) became more and more popular because of their higher efficiency and flexibility. A typical

1 Corresponding author.
CCPP is composed of a gas cycle and a steam cycle. The gas cycle is fed by fuel (usually natural gas) and produces electric power through the expansion of the gas in a (gas) turbine; the steam cycle is supplied with the exhaust gases from the gas turbine and generates both electricity and steam for other industrial processes like paper mills, chemical plants, etc. From the economic side, the liberalization of the energy market promoted the need of operating CCPPs in 
the most efficient way, that is by maximizing the profits due to the sales of steam and electricity and by minimizing the operating costs. Furthermore, due to the volatility of the electricity markets, flexibility of operation has become a "must". This fact makes lifetime issues relevant during normal operation. Indeed, only a detailed knowledge of the plant aging process can help to decide how aggressive the operational strategy can and should be in order to maximize profits.

In this paper we consider the problem of optimizing the short-term operation of a CCPP, i.e., to optimize the plant operation on an hourly basis over a time horizon that may vary from few hours to one day (Moslehi et al., 1991), while looking at the real cost of lifetime usage. A large stream of research in the power systems area focused on this problem. The usual recipe is to recast the economic optimization into the minimization of a cost functional and to account for the physical model of the plant through suitably defined constraints. See (Ferrari-Trecate et al., 2002b) and (Gallestey et al., 2001) for a list of related work.

In particular, we show how both the tasks of modelling and optimization of CCPPs can be efficiently solved by resorting to hybrid system methodologies. Hybrid systems have attracted the interest of many researchers, because they can capture in a single model the interaction between continuous and discrete-valued dynamics. Various models for hybrid system have been proposed (Labinaz et al., 1997), (Lygeros et al., 1999), (Branicky et al., 1998) and the research focused on the investigation of basic properties such as stability (Branicky, 1998), controllability and observability (Bemporad et al., 2000), and the development of control (Bemporad and Morari, 1999) (Lygeros et al., 1999), state estimation (FerrariTrecate et al., 2002a) and verification (Alur et al., 1996) schemes. We will use discrete-time hybrid systems in the Mixed Logical Dynamical (MLD) form (Bemporad and Morari, 1999) for two reasons: first, they provide a general framework for modelling many discrete features of CCPPs, including the coordination and prioritization between different devices; second, they are suitable to be used in on-line optimization schemes.

In Section 2 we briefly recall the basic features of MLD systems and in Section 3 we describe the CCPP plant we consider (the "Island" CCPP). In Section 3.1 it is shown how to model in the MLD form both the continuous and discrete features of the plant. Power plant lifetime modelling in the MLD framework will also be addressed. The operation optimization is then described in Section 4. Finally in Section 5 the most significant control experiments are illustrated.

\section{HYBRID SYSTEMS IN THE MLD FORM}

The derivation of the MLD form of a hybrid system involves basically three steps (Bemporad and Morari, 1999)

(1) The first one is to associate with a logical statement $S$, that can be either true or false, a binary variable $\delta \in\{0,1\}$ that is 1 if and only if the statement holds true. Then, the combination of elementary statements $S_{1}, \ldots, S_{q}$ into a compound statement via the Boolean operators AND $(\wedge)$, OR $(\vee)$, NOT $(\sim)$ can be represented as linear inequalities over the corresponding binary variables $\delta_{i}$, $i=1, \ldots, q$.

(2) The second step is to represent the products between bounded affine functions $f(x)$ and a logic variables by introducing auxiliary variables $z=\delta f(x)$. The value of $z$ can be equivalently assigned through mixed-integer linear inequalities.

(3) The third step is to include binary and auxiliary variables in an LTI discrete-time dynamic system in order to describe in a unified model the evolution of the continuous and logic components of the system.

The general MLD form of a hybrid system is (Bemporad and Morari, 1999)

$$
\begin{gathered}
x(t+1)=A x(t)+B_{1} u(t)+B_{2} \delta(t)+B_{3} z(t) \\
y(t)=C x(t)+D_{1} u(t)+D_{2} \delta(t)+D_{3} z(t) \\
E_{2} \delta(t)+E_{3} z(t) \leq E_{1} u(t)+E_{4} x(t)+E_{5}
\end{gathered}
$$

where $x=\left[\begin{array}{ll}x_{c}^{T} & x_{l}^{T}\end{array}\right]^{T} \in R^{n_{c}} \times\{0,1\}^{n_{l}}$ are the continuous and binary states, $u=\left[\begin{array}{ll}u_{c}^{T} & u_{l}^{T}\end{array}\right]^{T} \in$ $R^{m_{c}} \times\{0,1\}^{m_{l}}$ are the inputs, $y=\left[\begin{array}{ll}y_{c}^{T} & y_{l}^{T}\end{array}\right]^{T} \in$ $R^{p_{c}} \times\{0,1\}^{p_{l}}$ the outputs, and $\delta \in\{0,1\}^{r_{l}}$, $z \in R^{r_{c}}$ represent auxiliary binary and continuous variables, respectively. All constraints on the states, the inputs, the $z$ and $\delta$ variables are summarized in the inequalities (3).

MLD systems are a versatile framework to model various classes of systems. For a detailed description of such capabilities we defer the reader to (Bemporad and Morari, 1999), (Bemporad et al., 2000). In this paper, for the optimization of the plant we propose a predictive control scheme (Model Predictive Control - MPC) which is able to stabilize MLD systems on desired reference trajectories while fulfilling operating constraints.

\section{HYBRID MODEL OF A COMBINED CYCLE POWER PLANT}

The cogeneration combined cycle power plant Island consists of four main components: a gas turbine, a heat recovery steam generator, a steam turbine and a steam supply for a paper mill. 


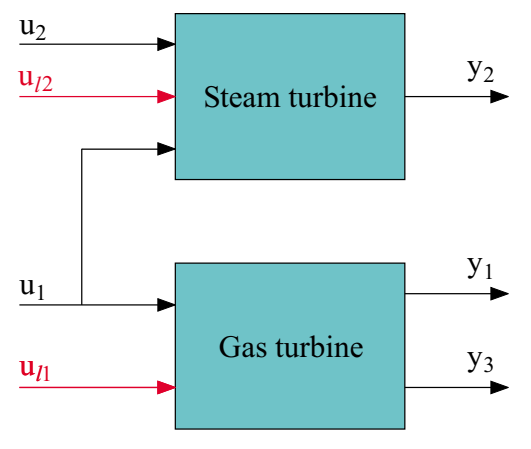

Fig. 1. Block diagram of the Island power plant.

We adopted the simplified input/output description of the plant presented in (Mossig, 2000) and represented in Figure 1. Note that the heat recovery steam generator does not appear in Figure 1 because it is hidden in the "steam turbine" block. The plant has two continuous-valued inputs $\left(u_{1}\right.$ and $\left.u_{2}\right)$, and two binary inputs $\left(u_{l 1}\right.$ and $\left.u_{l 2}\right)$ :

- $u_{1}$ is the set point for the gas turbine load (in percent). The permitted operation range for the gas turbine is in the interval $\left[u_{1, \min }, u_{1, \max }\right]$;

- $u_{2}$ is the steam mass flow to the paper mill. The permitted range for the steam flow is in the interval $\left[u_{2, \min }, u_{2, \max }\right]$;

- $u_{l 1}$ and $u_{l 2}$ are, respectively, the on/off commands for the gas and steam turbines; the "on" command is associated with the value one.

In the Island plant the inputs $u_{1}$ and $u_{2}$ are independent and all possible combinations within the admissible ranges are permitted. The binary input variables must fulfill the logic condition

$$
u_{l 2}=1 \quad \Rightarrow \quad u_{l 1}=1
$$

which defines a priority constraint between the two turbines: The steam turbine can be switched on/off only when the gas turbine is on, otherwise the steam turbine must be kept off.

The output variables of the model are:

- the fuel consumption of the gas turbine, $y_{1}$ $[\mathrm{kg} / \mathrm{s}]$

- the electric power generated by the steam turbine, $y_{2}[\mathrm{MW}]$;

- the electric power generated by the gas turbine, $y_{3}[\mathrm{MW}]$;

Since we aim at optimizing the plant hourly, we chose a sampling time of one hour and we assume that the inputs are constant within each sampling interval. As reported in (Mossig, 2000), a satisfactory input/output model of the plant is

$$
\begin{aligned}
& y_{1}(k+1)=f_{1}\left(u_{1}(k)\right) \\
& y_{2}(k+1)=f_{2}\left(u_{1}(k), u_{2}(k)\right) \\
& y_{3}(k+1)=f_{3}\left(u_{1}(k)\right)
\end{aligned}
$$

where the maps $f_{1}, f_{2}$ and $f_{3}$ can be either affine or piecewise affine and are obtained by interpolating experimental data. In particular, the use of piecewise affine input/output relations allows to approximate nonlinear behaviors in an accurate way.

Lifetime consumption in power plants is a huge field, where much activity is registered, see (Viswanathan, 1989) for an illustration. Given a power plant component (a turbine or compressor, but also parts at a lower level like pipes or blades) the useful lifetime is described by a function of time $L T:[0, \infty] \mapsto[0,1]$. By definition, $L T(t)=1$ indicates that the component is new and $L T(t)=0$ indicates that the component must be replaced or have an overhaul. For our purposes the time evolution of $L T$ can be described by a discrete time equation of the form

$$
\begin{aligned}
L T(k+1)= & L T(k)+f_{\text {on }}(u(k), L T(t))+ \\
& f_{\text {startup }}(u(k), L T(k))+ \\
& f_{\text {shutdown }}(u(k), L T(k))
\end{aligned}
$$

where

- $u$ is the turbine (gas or steam, as appropriate) load at time $k$,

- $f_{o n}$ is a non-positive function which is nonzero only if power is being produced. It describes the "wearing" due to constant operation and is equal to zero if $u(k)=0$.

- $f_{\text {startup }}$, respectively $f_{\text {shutdown }}$, denote nonpositive functions which are nonzero only when startup, respectively a shutdown, occurs at time $k$. These functions describe the damage caused by these operations.

The functions $f_{\text {on }}, f_{\text {startup }}$ and $f_{\text {shutdown }}$ are strongly nonlinear and are the result of elaborated tuning procedures considering the overall process conditions, temperature/stress distributions and material properties, see (Gallestey et al., 2001) for details and references.

\subsection{The MLD Model of the Island Plant}

The features which suggest modelling the Island power plant as a hybrid system are the following:

- the presence of the binary inputs $u_{l 1}$ and $u_{l 2}$;

- the turbines have different start up modes, depending on how long the turbines have been kept off;

- electric power, steam flow and fuel consumption are continuous valued quantities evolving with time.

- component lifetime is a continuous valued variable and, as is clear from (8), its decrease is partly due to startup and shutdown operation. 
Furthermore, the following constraints must be taken into account:

- the operating constraints on the minimum amount of time for which the turbines must be kept on/off (the so-called minimum up/down times);

- the priority constraint (4). This condition, together with the previous one, leads to constraints on the sequences of logic inputs which can be applied to the system;

- the gas turbine load $u_{1}$ and the steam mass flow $u_{2}$ are bounded.

Finally one would also like to describe the piecewise affine relations (5)-(7) and (8) in the model of the CCPP. All these features can be captured by hybrid models in the MLD form. Here, we only discuss how to model component lifetime in the MLD form and defer the reader to (FerrariTrecate et al., 2002b) for the modelling of each component.

Consider the following representation of (8),

$$
\begin{aligned}
L T(k+1)= & L T(k)+l t_{1}(u(k), L T(k)) \delta_{\text {on }}+ \\
& l t_{2}(u(k), L T(k)) \delta_{\text {startup }}+ \\
& l t_{3}(u(k), L T(k)) \delta_{\text {shutdown }},
\end{aligned}
$$

where the boolean variables $\delta$ denote the "producing power", "startup" and "shutdown" conditions, respectively. If the functions $l t_{1}, l t_{2}$ and $l t_{3}$ are accurate piecewise affine approximation of the true nonlinear behaviors, then, it is possible to represent the r.h.s. of (9) through a system of mixed-integer inequalities (Bemporad and Morari, 1999).

\section{PLANT OPTIMIZATION}

The control technique we use to optimize the operation of the Island power plant is Model Predictive Control (MPC). The main idea of MPC is to use a model of the plant (the MLD model in our case) to predict the future evolution of the system within a fixed prediction horizon of length $M$. Based on this prediction, at each time step $k$ the controller selects a sequence of future command inputs through an on-line optimization procedure, which aims at minimizing a suitable cost function, and enforces fulfillment of the constraints. Then, only the first sample of the optimal sequence is applied to the plant at time $k$ and at time $k+1$, the whole optimization procedure is repeated (Morari et al., 2001). In the case of economic optimization, the cost functional represents the operating costs minus the revenues obtained. One possible choice is

$$
\begin{aligned}
J & =C_{\text {dem }}+C_{\text {fuel }}+C_{\text {start up }}+ \\
& +C_{\text {fixed }}-E+C_{L T}
\end{aligned}
$$

where the terms $C_{\text {dem }}, C_{\text {start up }}, C_{\text {fuel }}$ and $C_{\text {fixed }}$ represent the costs and $E$ the earnings over the prediction horizon $M$, see (Ferrari-Trecate et al., $2002 b$ ) for further details. Below, we only discuss the term $C_{L T}$ related to lifetime consumption. $C_{L T}$ represents the asset value depreciation or lifetime usage cost due to a given operation strategy (i.e. the controls $u_{1}(\cdot), u_{2}(\cdot), u_{3}(\cdot)$, and $u_{4}(\cdot)$ ) over the predicton horizon $M$. Let $A V(k)$ denote the current asset value of the power plant at time $k$ that is given by

$$
A V(k)=\sum_{i=1}^{n c} V_{i} L T_{i}(k),
$$

where $n c, V_{i}$ and $L T_{i}(k)$ denote the number of relevant components, the corresponding proportionality factors, and the current lifetime (see (9)), respectively. Then, $C_{L T}$ is given by

$$
C_{L T}=A V(k)-A V(k+M-1) .
$$

\subsection{Derivation of the MILP}

The constraints of the optimization problem are the system dynamics (1)-(3) expressed in the MLD form. We denote with $f(t \mid k)$ a time function, defined for $t \geq k$, that depends also on the current instant $k$. Then, the overall optimization problem can be written as

$$
\begin{aligned}
& \min J \quad \text { subject to } \\
& x(k \mid k)=x_{k} \text { and for } t=k, \ldots, k+M \\
& x(t+1 \mid k)=A x(t \mid k)+B_{1} u(t \mid k)+B_{2} \delta(t \mid k)+ \\
& +B_{3} z(t \mid k) \\
& y(t \mid k)=C x(t \mid k)+D_{1} u(t \mid k)+D_{2} \delta(t \mid k)+ \\
& +D_{3} z(t \mid k) \\
& E_{2} \delta(t \mid k)+E_{3} z(t \mid k) \leq E_{1} u(t \mid k)+E_{4} x(t \mid k)+ \\
& +E_{5}
\end{aligned}
$$

where the state $x_{k}$ of the system at time $k$ enters through the constraint $x(k \mid k)=x_{k}$ and the optimization variables are $\{u(t \mid k)\}_{t=k}^{k+M-1}$, $\{\delta(t \mid k)\}_{t=k}^{k+M-1},\{z(t \mid k)\}_{t=k}^{k+M-1}$.

The optimization problem (13) is a mixed integer nonlinear program due to the nonlinearities appearing in the terms $C_{d e m}, C_{\text {change, }}, C_{\text {start up }}$, $E$, and $C_{L T}$. However these nonlinearities are piecewise affine maps and (13) can be re-written as a Mixed-Integer Linear Programming (MILP) problem by introducing auxiliary mixed-integer linear inequalities (Ferrari-Trecate et al., 2002b).

\section{CONTROL EXPERIMENTS}

In this section, we demonstrate the effectiveness of the proposed optimization procedure through some simulations. 
The input/output equations describing the plant are given by (5)-(7) where

$$
\begin{aligned}
f_{1}\left(u_{1}\right) & =0.0748 \cdot u_{1}+2.0563 \\
f_{2}\left(u_{1}, u_{2}\right) & =0.62 \cdot u_{1}-0.857 \cdot u_{2}+29.714 \\
f_{3}\left(u_{1}\right) & =1.83 \cdot u_{1}-0.0012
\end{aligned}
$$

The permitted range for $u_{1}$ and $u_{2}$ are summarized in Table 1. For the Island plant, the

\begin{tabular}{||l||l||l||}
\hline \hline Input & Minimum & Maximum \\
\hline \hline$u_{1}$ & $50 \%$ & $100 \%$ \\
\hline \hline$u_{2}$ & $2 \mathrm{~kg} / \mathrm{s}$ & $37 \mathrm{~kg} / \mathrm{s}$ \\
\hline \hline
\end{tabular}

Table 1. Upper and lower bounds on the inputs

affine models (14) and (16) are sufficiently accurate (Mossig, 2000), whereas equation (15) is a crude approximation of the nonlinear behavior. We highlight again the fact that a more precise MLD model could be obtained by using more accurate (and complex) piecewise affine approximations for the function $f_{2}$.

For a specified profile of the electric and steam demands, the optimizer chooses the optimal inputs in order to track the demands and at the same time minimize the operating costs. In particular, the behavior of the control action can be tuned by using suitable values of the weight coefficients appearing in the cost functional $J$. The turbines are switched on/off satisfying the operating constraints (the minimum up and down times and the different types of start up).

\subsection{Experiments with lifetime}

In (Ferrari-Trecate et al., 2002b) experimental results on power plant load optimization (but without lifetime terms in the cost functional) are discussed. For sake of brevity, here we only present experiments that illustrate the effect of including lifetime terms in the optimization functional.

The results are depicted in Figures 2 and 3. In the first experiment, $V_{i} \equiv 0$ for all the indices $i$ and then the term $C_{L T}$ is always null. In the second one, the magnitudes $V_{i}$ are positive for components related to the steam turbine (rotor, casing, blades, piping, etc.) and zero otherwise. In other words, we induce an optimizer behavior characterized by a "reluctancy" to use the steam turbine.

The pictures legend is

- continuous line: the electricity demand (MW),

- dashed line: sum of gas and steam turbine power output (MW),

- triangles: gas turbine power output (MW),

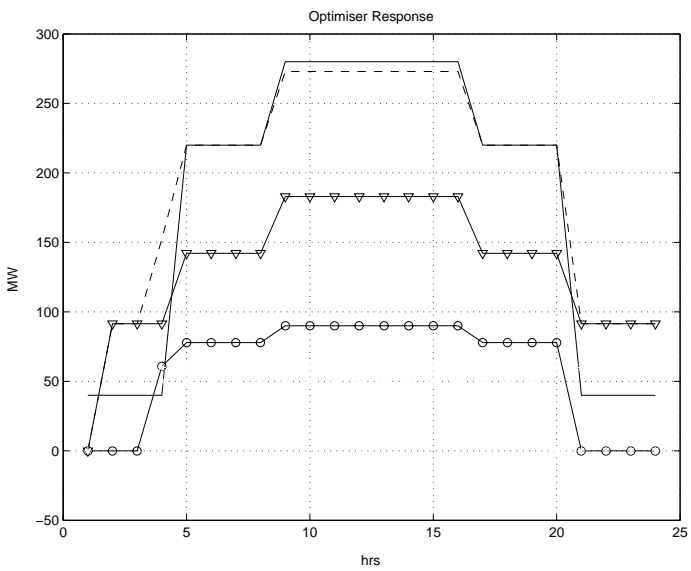

Fig. 2. Control experiment over one day with $M=7$ hours and $C_{L T} \equiv 0$.

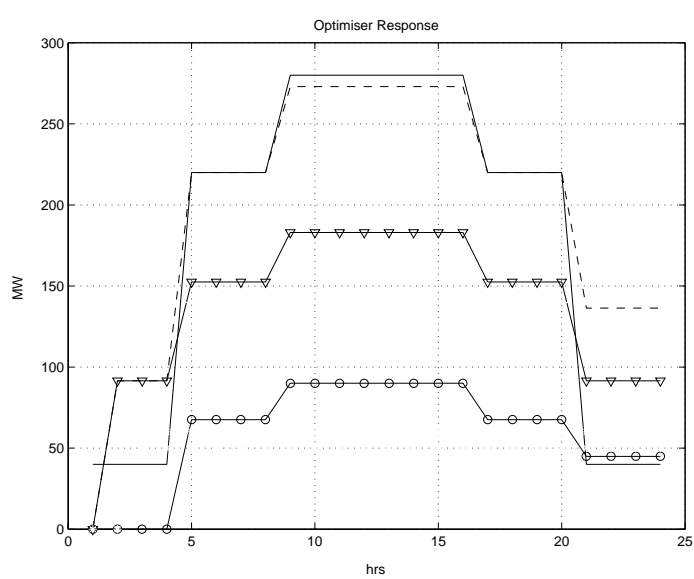

Fig. 3. Control experiment over one day with $M=7$ hours and $C_{L T}>0$ related to steam turbine lifetime consumption.

- circles: steam turbine power output (MW).

We note that, although the same overall power is produced, the task is distributed differently. Indeed, in the second case the steam turbine is started later and no shutdown takes place at the end of the period. Moreover, the power outputs differ significantly.

\section{CONCLUSIONS}

The main goal of this paper is to show that hybrid systems in the MLD form provide a suitable framework for modelling CCPPs. In particular, many features like the possibility of switching on/off the turbines, the presence of minimum up and down times, priority constraints between turbines, different startup procedures and component lifetime consumption can be captured by an MLD model. We point out that also other characteristics, like ramp constraints or nonlinear input/output relations (approximated by piecewise affine functions), can be easily incorporated in the MLD description. Then, the optimization 
of the operation can be recasted into an MPC problem that can be efficiently solved by resorting to MILP solvers.

In particular, we have introduced a novel approach to take into account the effect of a given operational mode on the asset depreciation, see also (Gallestey et al., 2001). Clearly, whether profits can be obtained via this method or not will depend largely on the plant characteristics, electricity and fuel prices, contractual constrains, etc. However, in authors opinion the idea of having lifetime explicitly in the functional to optimize is very powerful and should lead to large improvements in plant profitability.

Note that the economic factors we considered in the definition of the cost functional $J$ are not the only possible choices. Indeed, different piecewise affine terms reflecting other performance criteria could be added without changing the structure of the resulting optimization problem (FerrariTrecate et al., 2001). For instance, more sophisticated asset depreciation models due to plant aging, or the scheduling of several power plants with different properties and characteristics, like in the case of the unit commitment problem (Baldick, 1995), can be easily incorporated into the presented framework.

\section{REFERENCES}

Alur, R., T. A. Henzinger and P. H. Ho (1996). Automatic symbolic verification of embedded systems. IEEE Trans. on Software Engineering 22(3), 181-201.

Baldick, R. (1995). The generalized unit commitment problem. IEEE Transactions on Power Systems 10(1), 465-475.

Bemporad, A. and M. Morari (1999). Control of systems integrating logic, dynamics, and constraints. Automatica 35(3), 407-427.

Bemporad, A., G. Ferrari-Trecate and M. Morari (2000). Observability and Controllability of Piecewise Affine and Hybrid Systems. IEEE Trans. on Automatic Control 45(10), 18641876.

Branicky, M.S. (1998). Multiple Lyapunov functions and other analysis tools for switched and hybrid systems. IEEE Trans. on Automatic Control 43(4), 475-482.

Branicky, M.S., W.S. Borkar and S.K. Mitter (1998). A unified framework for hybrid control: model and optimal control theory. IEEE Trans. on Automatic Control 43(1), 31-45.

Ferrari-Trecate, G., D. Mignone and M. Morari (2002a). Moving horizon estimation for hybrid systems. IEEE Trans. on Automatic Control. to appear.

Ferrari-Trecate, G., E. Gallestey, P. Letizia, M. Spedicato, M. Morari and M. An- toine $(2002 b)$. Modelling and control of cogeneration power plants: A hybrid system approach. In: Proc. 5th International Workshop on Hybrid Systems: Computation and Control (C. Tomlin and M. Greenstreet, Eds.). Lecture Notes in Computer Science. SpringerVerlag.

Ferrari-Trecate, G., P. Letizia and M. Spedicato (2001). Optimization with piecewise-affine cost functions. Technical report. AUT00-13, Automatic Control Laboratory, ETH Zurich.

Gallestey, E., A. Stothert, M. Antoine and S. Morton (2001). Model predictive control and the optimisation of power plant load while considering lifetime consumption. IEEE Trans. on Power Systems. Accepted for publication.

Labinaz, G., M.M. Bayoumi and K. Rudie (1997). A Survey of Modeling and Control of Hybrid Systems. Annual Reviews of Control 21, 7992.

Lygeros, J., C. Tomlin and S. Sastry (1999). Controllers for reachability specifications for hybrid systems. Automatica 35(3), 349-370.

Morari, M., J. Lee and C. Garcia (2001). Model Predictive Control. Prentice Hall, Draft Manuscript.

Moslehi, K., M. Khadem, R. Bernal and G. Hernandez (1991). Optimization of multiplant cogeneration system operation including electric and steam networks. IEEE Trans. on Power Systems 6(2), 484-490.

Mossig, K. (2000). Load optimization. Technical report. ABB Corporate Research, Baden (Zurich).

Viswanathan, R. (1989). Damage Mechanisms and Life Assessment of High-Temperature Components. ASM International. 